\title{
Experimental and numerical investigation on the convective thermal plume around the head of the standing and lying hu- man body
}

\author{
Laurențiu Tăcutu ${ }^{1}$, Ilinca Năstase $^{1, *}$, Florin Bode ${ }^{1,2}$, Cristiana Verona Croitoru', Angel Dogeanu ${ }^{1}$, and Mihnea \\ Sandu ${ }^{1}$ \\ ${ }^{1}$ CAMBI research center, Technical University of Civil Engineering, Bucharest, Romania \\ ${ }^{2}$ Technical University of Cluj-Napoca, Cluj-Napoca, Romania
}

\begin{abstract}
This paper presents a study for two thermal plumes generated by two humanoid thermal manikins, one standing and one lying down. The research was approached from a numerical and experimental perspective. The numerical model represents an operating room (OR) with two surgeons, a patient and a unidirectional air flow (UAF) diffuser. The experimental study was made in a climatic chamber, having a similar air distribution system, using particle image velocimetry (PIV) and infrared thermography (IR) measurements. The purpose of the study was to characterize the thermal plumes of the two manikins by numerical and experimental studies. The results obtained from these different approaches were compared with each other and with the ones from the literature in order to validate our numerical models.
\end{abstract}

\section{Introduction}

A typical problem regarding the conception of building systems and simulation of the resulting indoor environment, which is still not acknowledged, are the convection flows caused by heat sources, like the human body plume. These thermal plumes can significantly affect the flow distribution in rooms, especially when significant heat sources (occupants, equipment's) are present in the enclosure[1-3]. The existence of such a scenario with the presence of a ventilation system that have low velocities at the terminal units can be found in practice, usually in old ORs. Generally, attention is given only on the flow generated by the air diffusion terminal devices. As shown by [4] the point of occurrence of the maximum air velocity in the occupied zone depends on the heat source strength and its distribution in the room. This type of situation is encountered in practice. Thus, the air flows interaction itself is of great importance when estimating occupants' comfort or pollutant dispersion. In the same time, results obtained from computational fluid dynamics (CFD) need to be validated with experimental data from real scale measurements before using CFD for larger parametric investigation. Thermal plumes of the human body have been studied during time from different approaches[1-3,5]. This paper presents a study on the thermal plumes generated from two humanoid thermal manikins, in an operating room (OR) scenario, from a numerical and experimental approach. The two manikins in this study were used in the position of a surgeon (standing position) and in the position of a patient (lying down position). Both manikins had the surface temperatures of a normal human. The hands of the surgeon were placed above the patient, in an operating scenario that is commonly found in practice.

\section{Methods}

The measurements were made in a climatic chamber that simulates a real scale OR, which has the dimensions of $3.5 \times 3.5 \times 2.5 \mathrm{~m}(1 \times L \times H)$, a hydraulic and automation system for temperature control on each wall, including door, and an air ventilation system with a UAF diffuser, also called as laminar air flow (LAF) diffuser. This study was performed in isothermal conditions $\left(20^{\circ} \mathrm{C}\right)$ for both cases, experimental and numerical. Two humanoid thermal manikins were used to simulate the natural convection flow from two human bodies. The scenario of the OR, with the scheme of the numerical case can be seen in Fig 1.

The surface temperature used for the manikins were Oarms $=30^{\circ} \mathrm{C}$, Olegs $=27^{\circ} \mathrm{C}$, Ocore $=32^{\circ} \mathrm{C}$, Ohead $=$ $34^{\circ} \mathrm{C}$. For determining the velocity vectors of the thermal plumes, a classical PIV system was used. A sketch with the equipment used and their placement can be seen in Fig. 2 (1 - CMOS camera, 2 - double pulsed laser, 3 laser unit, 4 - multiplexer, 5 - external command panel, 6 - PC, 7 - smoke generator, 8 - compressor, 9 - manikin,

\footnotetext{
* Corresponding author: ilinca.nastase@utcb.ro
} 
10 - UAF). For measuring the gradient temperature of the manikins, a black cardboard with a grid of metallic dots place at a distance of $50 \mathrm{~mm}$ between there axis was made and used.

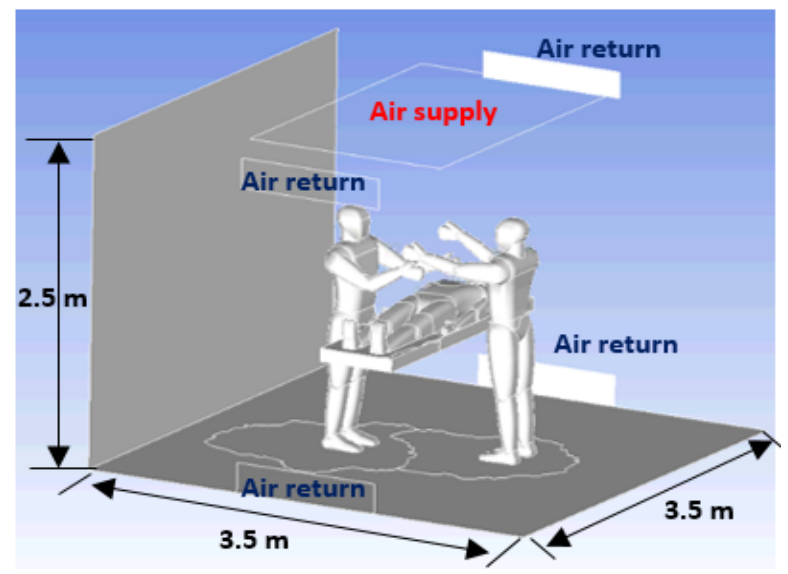

Fig. 1. OR scheme with dimensions, manikins and ventilation system.

a)

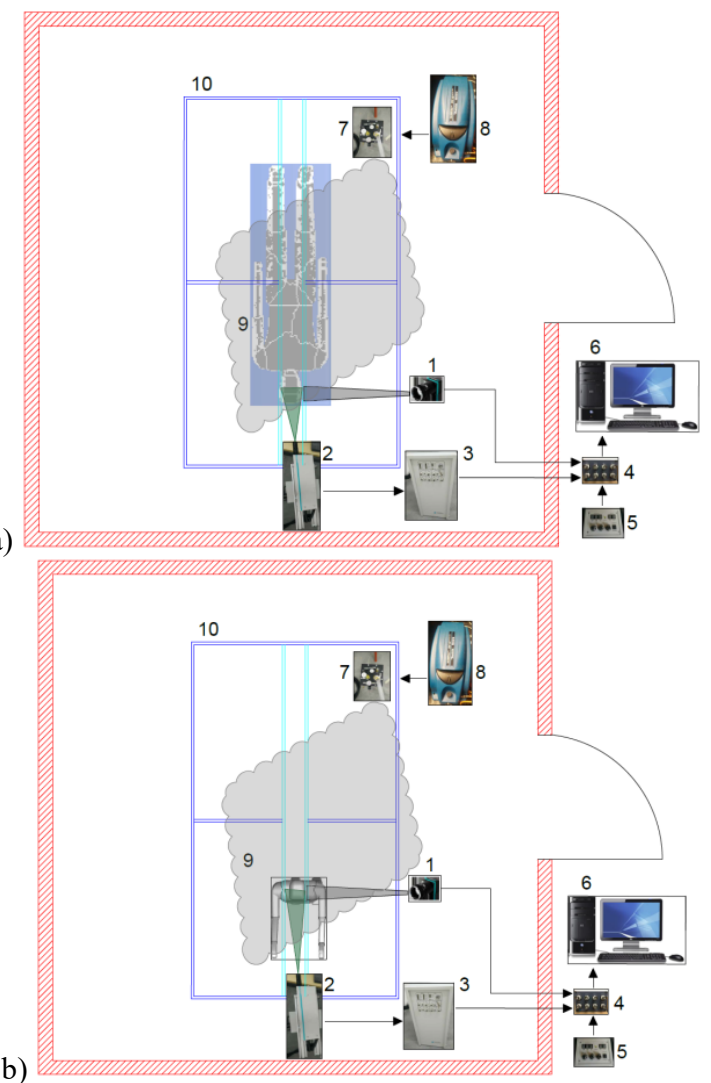

Fig. 2. Equipment placement for the PIV measurements: a) patient thermal plume; b) surgeon thermal plume.

The numerical model was made in Ansys Workbench 18.2, using Design Modeler for making the geometry and Fluent for calculating the equations. Two numerical cases were made. The first numeric case consists only with the patient on the bed, while the second numerical case consists in two surgeons and the patient that lies on a bed (Fig. 1). The bodies of all the occupants have an anatomic shape. The virtual manikin has a height of $1.75 \mathrm{~m}$ and a body surface of $1.8 \mathrm{~m}^{2}$. The computational grid for the initial case is composed of 3.6 million of tetrahedral elements, while for the second case is composed of 18.3 million of tetrahedral elements. The boundary layer for the manikins consists of 8 layers, with a grow of 1.2. For both cases, grid independency test was made, testing 10, 11.8, 13.75, 18.3, 20.3 milion of tetrahedral elements for the case with two surgeons and 1.3, 2.5, 3.6, 4.4 milion of tetrahedral elements for the case with the patient. Because the shape of the manikins is reproducing a human shape in large details, ignoring the fine details like eyes, eyebrows, nails, and so on, the size of the mesh tends to increase significant. However, the use of a humanoid manikin allows a more realistic approach of the study and a deeper understanding of the phenomenon studied.

a)

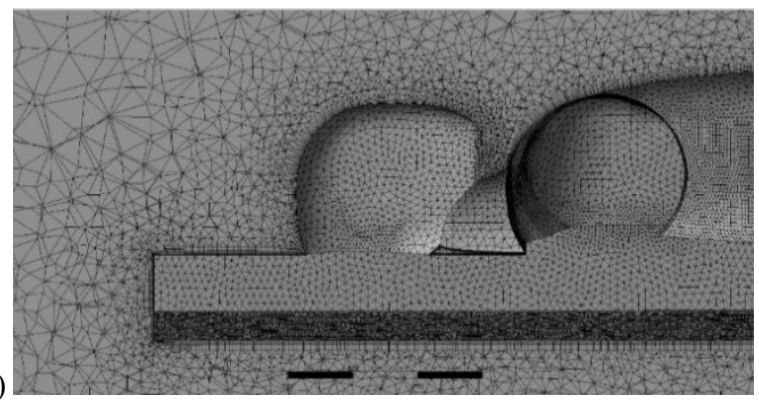

b)

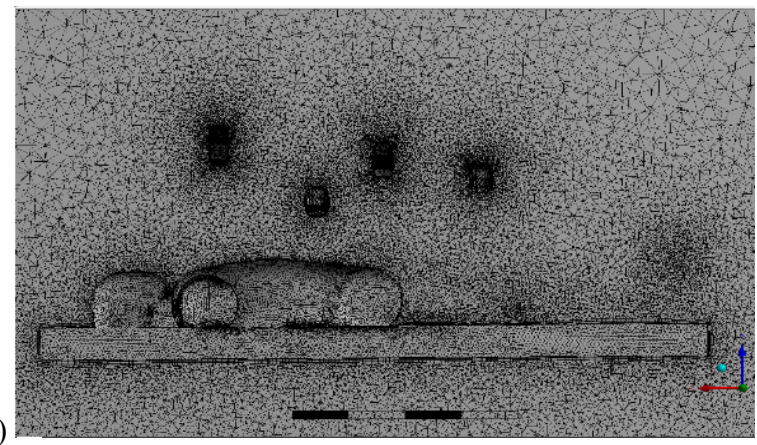

Fig. 3. Mesh section with the interest domain: a) patient; b) patient and surgeon's hands.

Walls and manikin's temperature were set as boundary conditions. Initially, laminar turbulence model was used with coupled scheme, for pressure velocity coupling, and hybrid initialization. Future studies were made with k- $\omega$ SST as for turbulence model, as it has been shown in previous studies that it is a more propitious model for studying such phenomena[6-9].

\section{Results}

Temperature and velocity measurements in the sagittal planes of the manikins were performed and compared between the numeric and experimental data. The numerical case with the two surgeons was calculated, initially for testing, only with one surgeon and the patient active (with human surface temperatures), while the second surgeon had a body temperature $20^{\circ} \mathrm{C}$ (Fig. 4). 


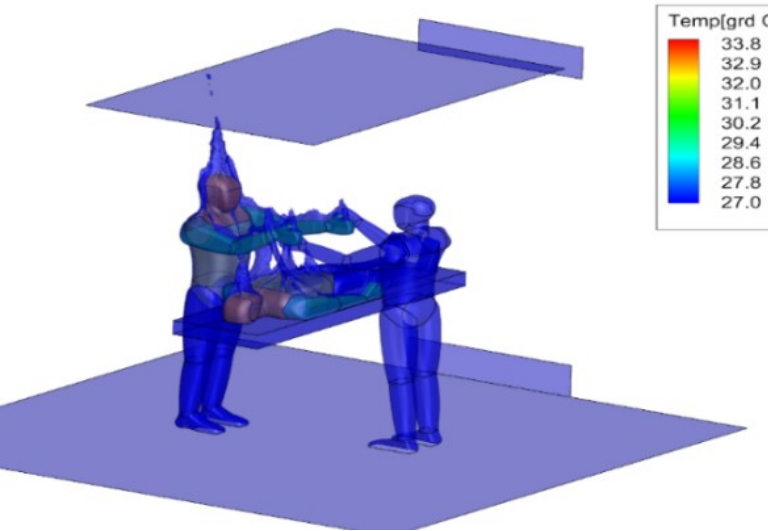

Fig. 4. 3D view of the temperature iso-surfaces from one surgeon and the patient in the OR.

Velocity vectors were obtained by post-processing the data from PIV measurements using the equipment software. An image mask was made in order to avoid bad vectors like the vectors behind the head, were the laser beam was covered. For post processing, adaptive correlation and vector statistics analyses were made to determine the velocity profiles (Fig. 5).

a)

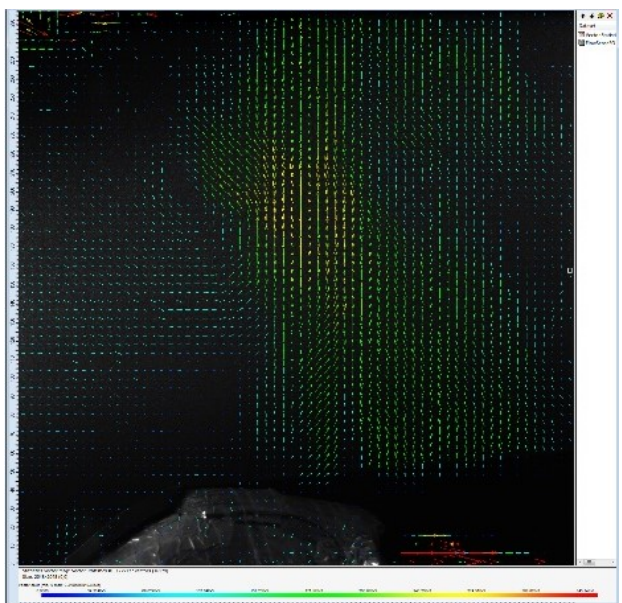

b)

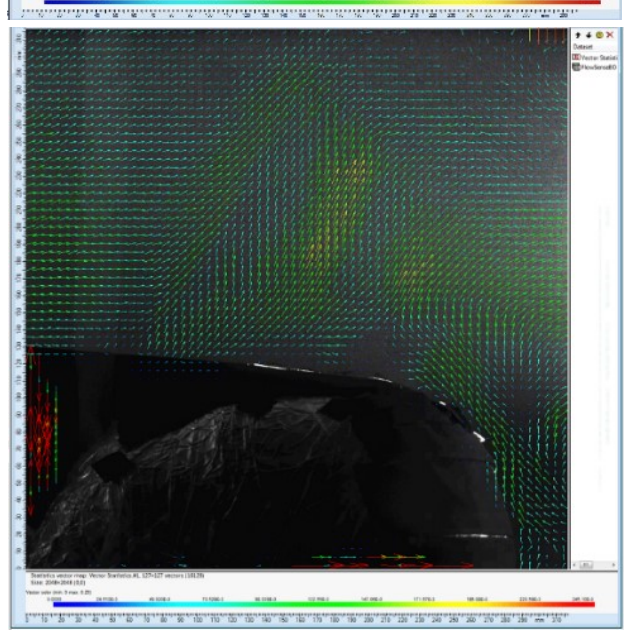

Fig. 5. Thermal plume velocity vectors from PIV software: a) surgeon; b) patient.

The gradient temperature obtained for the surgeon, from both approaches, have resulted to have almost the same allure and the same covered height for identical temperature range $\left(\approx 24 \div 34^{\circ} \mathrm{C}\right)$. In this case the temperature gradient has almost the same allure in both approaches. The distance between the dots on the IR measurements are $50 \mathrm{~mm}$ on vertical and horizontal.

From both approaches it was determining that the velocity vectors of the surgeon can have a peak around $0.35 \mathrm{~m} / \mathrm{s}$ above the head, at a distance of $\approx 80 \div 150 \mathrm{~mm}$. In the numerical case, peak velocities were observed forming also in the uppermost part of the lateral parts of the head.Regarding the patient thermal plume, the temperature gradient and the velocity vectors from the measurements showed a smaller height than the one of the surgeons. The temperature gradient height covered $50 \div 100 \mathrm{~mm}$ at most. Also, from the numerical case of the patient it was observe the same phenomenon as in the numerical case of the surgeon, regarding the convective boundary layer.

a)

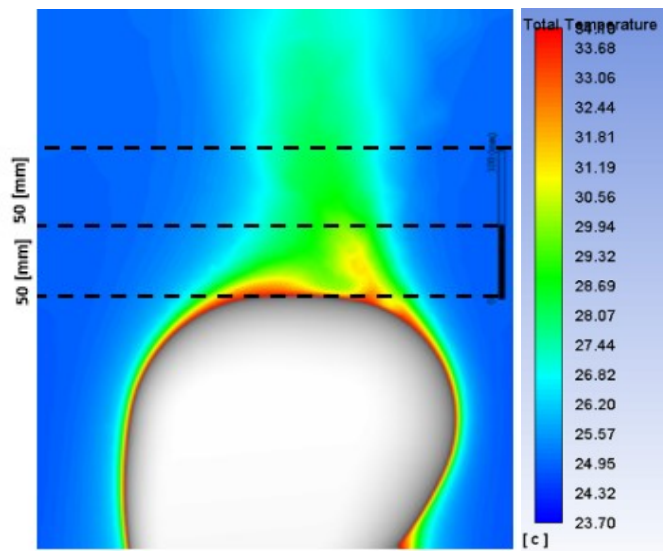

b)

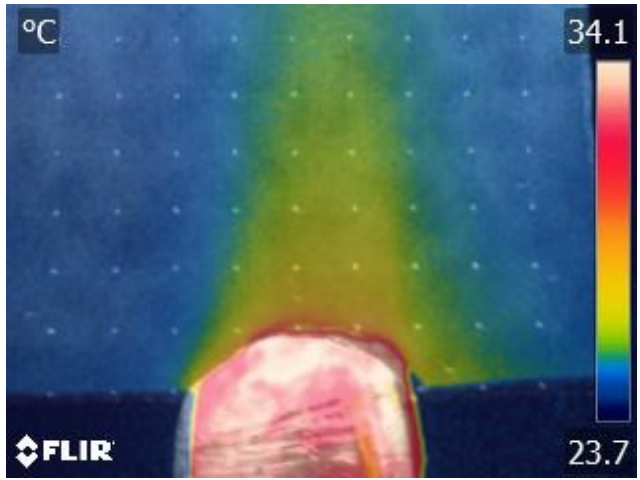

Fig. 6. Surgeon thermal plume gradient temperature: a) CFD; b) IR measurements.

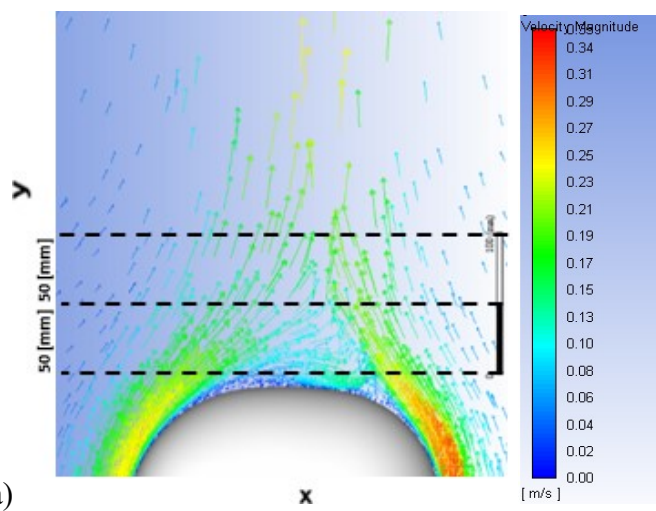


b)

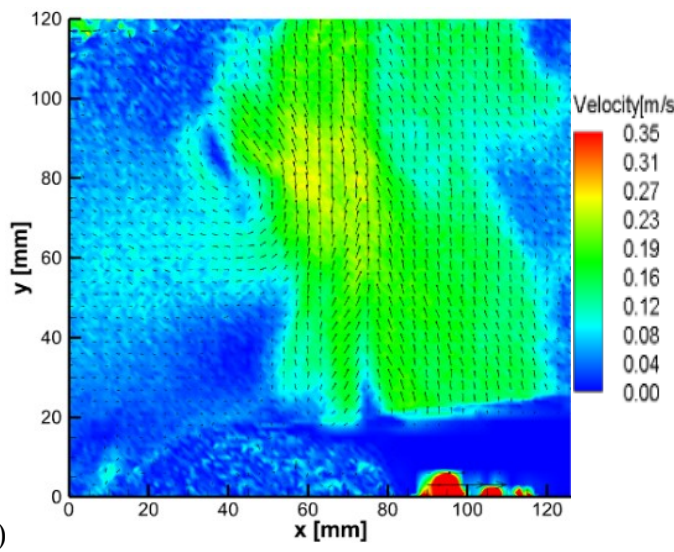

Fig. 7. Thermal plume velocity vectors of the surgeon: a) CFD; b) PIV measurements.

a)

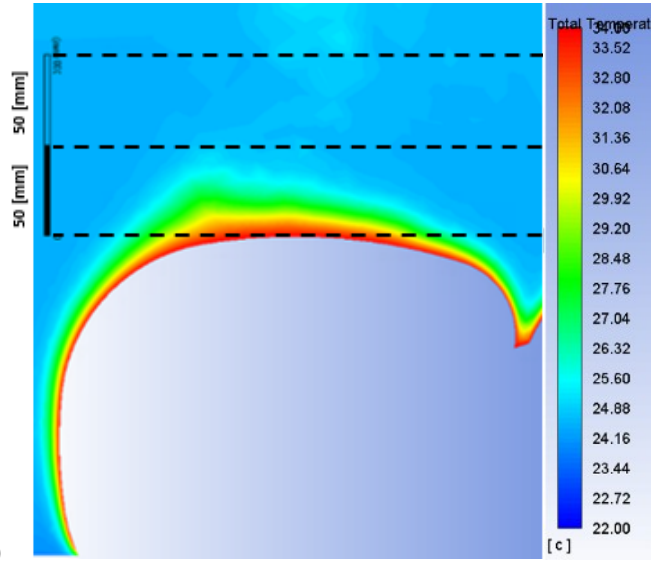

b)

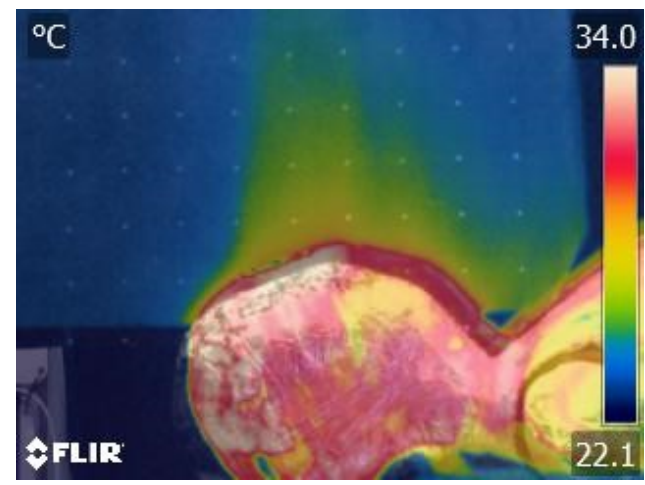

Fig. 8. Patient thermal plume gradient temperature: a) CFD; b) IR measurements.

a)

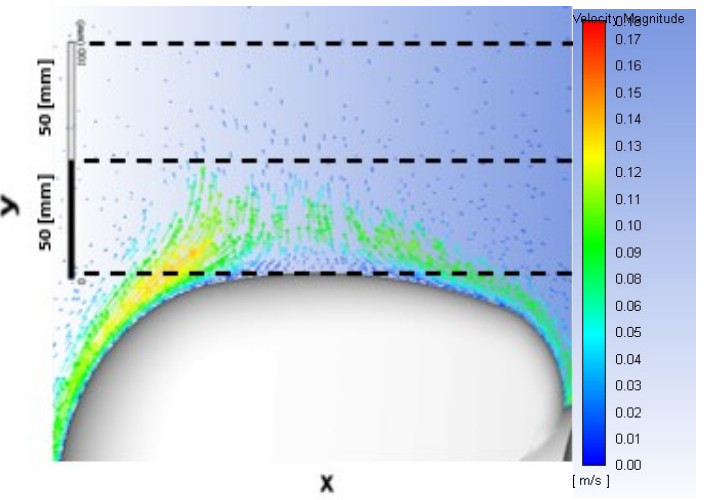

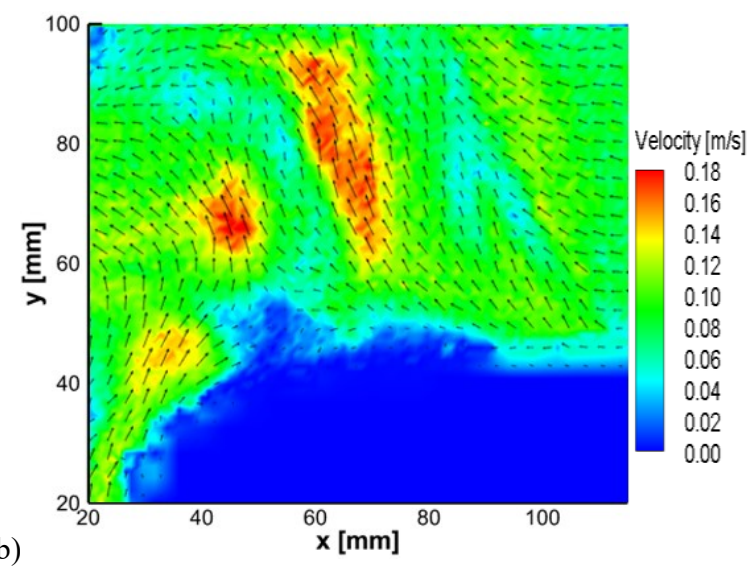

Fig. 9 Patient thermal plume velocity vectors:

a) CFD; b) PIV measurements.

Patient velocity vectors have showed a peak of $0.18 \div 0.20 \mathrm{~m} / \mathrm{s}$ resulted at $50 \mathrm{~mm}$ above the head. For patient, the velocity vectors were much lower than the ones of the surgeon. An observation that can be seen in both numerical cases, patient and surgeon, for both parameters (temperature and velocity), is the gradual increase of the convective boundary layer on the sides of the manikins, near the head.

\section{Conclusions}

The importance of studying thermal plumes comes from their effect on the air circulation in an enclosure, which automatically has repercussion on the thermal comfort, aerosol distribution and energy efficiency. These influences can be significant for displacement ventilation systems, where the velocities of the inlet air are lower. The results showed in this paper come to validate other studies, were research was made $[1,10,11]$, and showed good correlation between the data obtained from numerical and experimental studies, regarding ranges and some similarities, regarding the distribution.

It has been showed in this study that the thermal plume from a human with a static to moderate activity can reach velocities up to $0.4 \mathrm{~m} / \mathrm{s}$, if the human body has a standing position (surgeon), and up to $0.2 \mathrm{~m} / \mathrm{s}$ seen above the head of the human body if standing in a lying down position on a bed(patient), both with the bodies uncovered. Bringing into discussion that in this range of values $(0.2 \div 0.4 \mathrm{~m} / \mathrm{s})$ is supplied the air from a UAF diffuser in a OR, we can see that studying this field can lead to actions meant to improve the air distribution[12].

The results showed that for a human that lies down in a supine position, the peak velocity is at around $20 \div 40 \mathrm{~mm}$ above the head, while the peak velocity of a standing human can be at $40 \div 60 \mathrm{~mm}$ above the head. Also, from this study it can be seen that the height of the thermal plume for a human that is standing can have a height of $100 \div 150 \mathrm{~mm}$, while for the one that is lying downhas $\approx 50 \mathrm{~mm}$ in the head region. 
Another observation regards the natural convective boundary layer which tends to form around the head and has an upward trajectory that can have a velocity increase at the breaking point from the surface. In the upward trajectory the boundary layer tends to grow in thickness and velocity.

This research field should be further studied because it presents many scenarios that have not been or are not fully researched and because it has influences on many factors like thermal comfort, ventilation and air distribution systems, energy efficiency and so on.

This work was supported by a grant of the Romanian space agency ROSA, QUEST - Advanced air diffusion system of the crew quarters for the ISS and deep space habitation systems, STAR-CDI-C3-2016-577.

\section{References}

1. Yang C., Yang X., and Zhao B., The ventilation needed to control thermal plume and particle dispersion from manikins in a unidirectional ventilated protective isolation room. Building Simulation, 2015. 8(5): p. 551-565.

2. Devienne R., et al., Experimental characterization of a plume of passive contaminant above a thermal source: capture efficiency of a fume extraction hood. Annals of Occupational Hygiene, 2009. 53(7): p. 739-48.

3. Zhou S. Q. and Xia K. Q., Plume statistics in thermal turbulence: mixing of an active scalar. Physical Review Letters, 2002. 89(18).

4. Kosonen, R., et al., Impact of heat load location and strength on air flow pattern with a passive chilled beam system Energy and Buildings, 2010. 42(1): p. 34-42.

5. Melikov A. and Kaczmarczyk J., Measurement and prediction of indoor air quality using a breathing thermal manikin. Indoor Air, 2007. 17(1): p. 50-9.

6. Bode, F., et al., A Numerical Analysis of the Air Distribution System for the Ventilation of the Crew Quarters on board of the International Space Station. E3S Web Conf., 2018. 32: p. 01006.

7. Bode, F., et al., Preliminary Numerical Studies for the Improvement of the Ventilation System of the Crew Quarters on Board of the International Space Station. INCAS BULLETIN, 2018.

8. Meslem, A., et al., Comparison of turbulence models in simulating jet flow from a crossshaped orifice. European Journal of Mechanics B/Fluids, 2014. 44: p. 100-120.

9. Meslem, A., et al., Optimization of Lobed Perforated Panel Diffuser: Numerical Study of Orifice Geometry. Vol. 6. 2012.

10. Clark R.P. and Edholm O.G., Man and his thermal environment. 1985, London: E. Arnold.

11. Clark R.P. and Toy N., Natural convection around the human head. Journal of Physiology, 1975. 244: p. 283-293.

12. Cao, G., et al., Do surgeons and surgical facilities disturb the clean air distribution close to a surgical patient in an orthopedic operating room with laminar airflow? American Journal of Infection Control, 2018. 46(10): p. 1115-1122. 